J Androl Gynaecol

September 2020 Vol::8, Issue:2

(c) All rights are reserved by Huang WL.

\section{How to treat Urinary Incon- tinence in Women without Surgery?}

Keywords: Urinary incontinence; Surgery; Traditional Chinese medicine; Acupuncture; Energy; Diet; Moxibustion; Homeopathy; Crystal-based medications; Chakras

\begin{abstract}
Statement of the problem: Urinary incontinence is defined as the loss of bladder control. In Western medicine, the treatment is hard to achieve and the need of surgery is common. In traditional Chinese medicine (TCM), urinary incontinence is caused by Kidney-Yang deficiency.

Purpose: To demonstrate it is possible to treat urinary incontinence without the need of surgery.

Methods: A case report of 55-years-old female patient, with urinary incontinence for around 30 years. After three years of the birth of her third and last child, all delivered by vaginal births, the symptoms appeared when she would sneeze or cough and worsened, requiring diapers for performing different activities, mainly when she had contact with water in her feet. Her gynecologist recommended her for surgery, but she refused for a long time and after years, she tried another treatment. She was diagnosed with Kidney-Yang deficiency and Blood deficiency according to TCM. She performed the procedure of radiesthesia, which resulted in level 1 of 8 in energy, the minimum possible. She started Chinese dietary therapy, auricular acupuncture apex ear bloodletting, moxibustion.
\end{abstract}

Findings: She had complete improvement of the symptoms in three Chinese dietary therapy, sessions of acupuncture, homeopathy and crystal-based medication, not needing to use diapers or sanitary pats anymore.

Conclusion: Based on this case report, we conclude that it is possible to treat urinary incontinence without surgery. To this aim we need to see the patient as a whole, considering the energy imbalances, which were causing urinary incontinence, using Chinese nutritional therapy, acupuncture, moxibustion, homeopathy and crystal-based medications.

\section{Introduction}

Urinary incontinence is defined as the loss of bladder control. The main symptoms are to lose urine when sneezing or coughing, but the problem can become more serious. Despites being commonly associated with aging and traumatic childbirth, there are many causes of urinary incontinence, and many different solutions and treatments [1-3].

The incontinence has several levels and can be associated with diuretic liquids in temporary cases, but it can also be permanent and constant during pregnancy, after childbirth or with aging [1-3].

Childbirth is believed to be one of the main causes of urinary incontinence. Women who delivered by caesarean sections have less risk of having urinary incontinence, even though the problem is also very common for those that did not have vaginal births. In a research performed by Yongtae Kim, which evaluated 15,307 women, $21 \%$ of those who had vaginal births had long-term urinary incontinence,
Journal of

\section{Andrology \& Gynaecology}

\section{Huang $W^{*}$}

Medical Acupuncture and Pain Management Clinic, Brazil

\begin{abstract}
*Address for Correspondence
Ling HW, Infectious Disease Specialist, General Practice, Nutrition Doctor, Acupuncture, Pain Management Specialist, Medical Acupuncture and Pain Management Clinic, Rua Homero Pacheco Alves, 1929, Franca, Sao Paulo, 14400-010, Brazil; E-mail: weilingmg@gmail.com

Submission: 21 August, 2020

Accepted: 18 September, 2020

Published: 22 September, 2020

Copyright: $\odot 2020$ Huang WL. This is an open access article distributed under the Creative Commons Attribution License, which permits unrestricted use, distribution, and reproduction in any medium, provided the original work is properly cited.
\end{abstract}

comparing with $16 \%$ of those who had caesarean sections [4].

Other studies showed demonstrated that despite the easiness in diagnosing the disease, a successful treatment is hard to achieve. The prevention is complex, maybe because there are other unknown aspects linked to the pathology, not recognized by current Western medicine [1-3].

Even with the exercises and other treatments available, the necessity of surgery is common. Therefore, there are doctors that would recommend the preference for caesarian sections in order to try to avoid the problem [5].

According to the article entitled Long-term Results of the TensionFree Vaginal Tape (TVT) Procedure for Surgical Treatment of Female Stress Urinary Incontinence, the urinary incontinence was completely solved in $56 \%$ of the cases [6].

The annual cost for women with urinary incontinence in the United States is around $\$ 12.4$ billion, with the average woman spending about $\$ 900$ each year to deal with the effects of bladder leakage [7].

\section{Purpose}

Demonstrate another way of treating for urinary incontinence, less aggressive then surgery using acupuncture and other principles of traditional Chinese medicine.

\section{Methods}

The method used in this article was to analyze the literature available in Western and traditional Chinese medicine, along with the use of a case report of a 55 years-old patient, E.M.P, married at 23, with three kids, all them delivered by vaginal births.

The problem started only when she would sneeze or cough. Within the years the conditions got a lot worse, until the moment she had to use diapers to perform many activities, especially those involving water.

When she first started to look for treatment for the condition, it was already unbearable. Talking to a gynecologist, she was willing to do the surgery for recovery of the condition. On the same week, she 
started treatment with the author. At first, she was diagnosed with Kidney-Yang deficiency and Blood deficiency according to TCM. After, it was started a treatment for her energy imbalances, first with Chinese dietary counseling. The diet recommended was to avoid dairy products, cold beverages (including water), raw food, and sweets. It was also recommended to avoid coffee, soda and matte tea. She also started twice a week sessions of auricular acupuncture and apex ear bloodletting.

\section{Results}

After two sessions, the patient started feeling results, within a month she obtained complete cure of the condition, not requiring the use of surgery.

\section{Discussion}

The urinary incontinence is a widespread problem among women, especially those who had several vaginal births in a short period of time [4].

The surgery also harms the routine of the patient, the complications associated with the surgery are also to be considered. On Hippocrates oath, it is stated "I will not use the knife, not even on sufferers from stone, but will withdraw in favor of such men as are engaged in this work", meaning that it is important to try to treat any disease clinically is a better option than performing a surgery, when possible [8].

Even though the surgery present good results, the surgical environment may not be the best recommendation according to Western medicine, the nosocomial environment is believed to increase the risk of nosocomial infection and other possible complications [9].

Due to these factors, many women search for different ways of treatment and cure, and many times the solution can be found in other ancient traditions, as traditional Chinese medicine.

\section{Western medicine and urinary incontinence}

There are three types of urinary incontinence. The first one is the SUI or Stress Urinary Incontinence; this type is characterized by the weakness of pelvic muscles. The main way of cure for SUI is exercises in order to fortify the pelvic muscles. These exercises are entitled Kegel exercises [1-6].

The second type is the Overactive Bladder or OAB, also called urgency incontinence. The process of this type of incontinence is miss signal sent by the brain, which understands that the bladder is needs to be empty, when it is not completely full yet. The main consequence is the lack of control of bladder every time the urge to urinate appears [1-6].

The third type is the mixed incontinence, which happens when the patient has both SUI and OAB. The patient described in this case report had mixed incontinence [1-6].

In Western medicine, therapy for urinary incontinence includes behavioral therapy, physiotherapy (such as Kegel and other similar exercises), medications and surgery. Duloxetine is the first licensed drug for the treatment of urinary incontinence, with rates of success around $66 \%$ [1-6].

According to the National Association of Incontinence, the
Women's Health Organization and the American Medical Systems, nowadays, 25 million adult Americans suffer from some level of urinary incontinence, and from those $75-80 \%$ are women [10].

The problem affects 200 million people worldwide. One in every four women over the age 18 experience episodes of urinary incontinence, even when the problem is not persistent. The diagnosis takes a long a time, and the possibility of surgery only exists when the woman completely lost the bladder control. Around the world, urinary incontinence is twice as common in women as in men. Pregnancy, childbirth and menopause are the major causes of incontinence in women. Between the ages of 18 to $44,24 \%$ of women will experience incontinence. For women over age $60,23 \%$ deal with the problem daily [10].

\section{Traditional Chinese medicine and the causes of urinary incontinence}

In the last decade, many studies aim to certificate the effectiveness of traditional Chinese medicine in the most varied pathologies. For treating asthma, allergies and also urinary incontinence, the principles of traditional Chinese medicine shown themselves as effective ways of treatment, and their use has been increasing $[11,12]$.

The main idea here, is to understand how Western and traditional Chinese medicines only see the symptoms and signs from two different perspectives. In the treatment of urinary incontinence, as TCM aims to see the patient as a whole, the first important step for treatment is an analysis of the patient historic. For example, a woman who gave birth to three babies, as the patient being described in this case report, will be more vulnerable to the urinary incontinence due to the deficiency of energy of the Kidney and Spleen leading to Blood deficiency and Yang deficiency resulting in Qi deficiency [13].

These energy deficiencies are well explained in the way postpartum period is interpreted, and how women should behave in this period [13].

Kidney and urinary bladder: Yin and Yang and Five Elements theory: In the Yellow Emperor's Classic of Internal Medicine, it is stated that a good physician, in order to perform a diagnosis of any pathologies has to analyze the symptoms and classify them into Yin and Yang in order to perform the diagnosis. The Bladder, according to the Five Elements theory is the hollow organ related to the Kidneys meridian or massive organ [14].

The Kidney meridian is linked to the Water element and the emotion of fear. On the same way, Kidney imbalanced can generate fear as well. When there is a Kidney-Yang deficiency, the Bladder meridian may suffer, what may reflect as urinary incontinence [13].

The bladder prolapse in Western medicine is associated with the weakening of the pelvic floor, due to vaginal birth. However, patients with performed caesarian session also develop urinary incontinence, as well as patients who did not bear children. On traditional Chinese medicine, the Yang energy is associated with the ability of keeping the internal organs "up", or on their proper place. When there is prolapse (of bladder, rectum or uterus), this is a symptom associated with Kidney-Yang deficiency, as it is possible to see on (Figure 1).

The second theory of major importance for traditional Chinese 


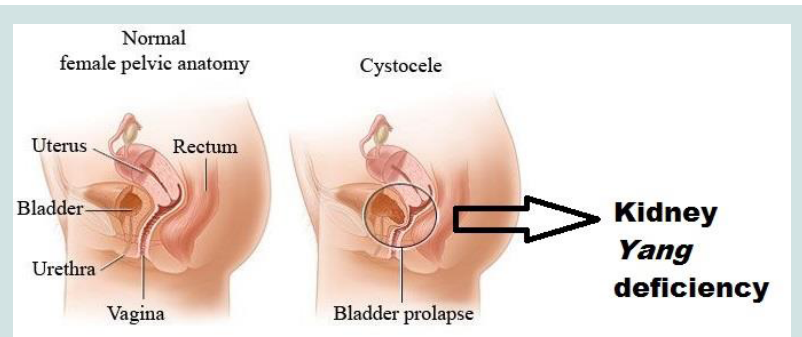

Figure 1: Bladder Prolapse and Kidney-Yang deficiency.

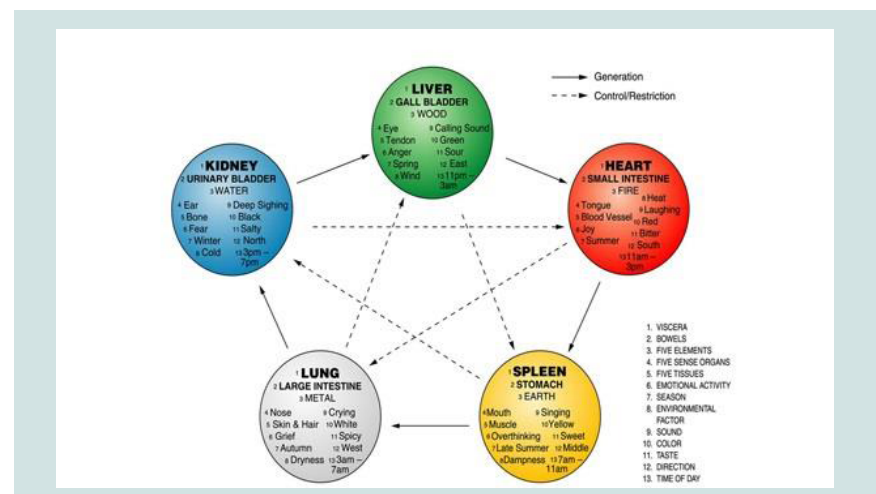

Figure 2: Organs classifications based on the five element theory.

medicine, together with the Yin and Yang theory is the Five Elements theory. The main objective of this theory is to establish relationship between the organs, comprehending the patient as whole [15].

In the case of urinary incontinence, the childbirth process and the loss of energy due to aging can have a negative influence over the body, causing the pathology. When the Five Elements scheme is comprehended (Figure 2), the physician will finally be able to see the body as whole, with interconnections between the body, receiving external and internal influence, and, with this knowledge in hands, it will become easier to develop new ways of treatment.

Post-partum period: According to traditional Chinese medicine, during the birth, there is an exceptional use of energy. A major part of vital energy, $Q i$ and Yang energy will be applied to bring the baby to the world. The postpartum therefore, is the moment where their energies would be more weakened, and their body would be fragile, being opened to bad energies, emotional and elemental, especially cold and wind. In order to conserve $Q i$, the patient is oriented to not force her eyes and mind. Not engaging in stressful mental activities. It is also common the orientation to sleep and rest a lot, being able to heal and become strong again [16].

Table 1: How to Diagnose Yin, Yang, Qi, Blood and Heat Retention Imbalances?.

\begin{tabular}{|c|c|c|c|c|c|}
\hline Question & $\begin{array}{c}\text { Does the } \\
\text { patient have } \\
\text { a daily bowel } \\
\text { movement? }\end{array}$ & $\begin{array}{c}\text { Does the patient } \\
\text { have excessive } \\
\text { sweating during } \\
\text { day? }\end{array}$ & $\begin{array}{c}\text { Does the patient feel cold in } \\
\text { the extremities of the body? } \\
\text { (Cold feet or hands) }\end{array}$ & $\begin{array}{c}\text { Does the patient } \\
\text { feel hot in the } \\
\text { extremities? }\end{array}$ & $\begin{array}{c}\text { Does the patient has dry mouth, bleeding gums, } \\
\text { bad breath, acne and/or redness in the skin, } \\
\text { abdominal pain, micro hematuria, or itching? }\end{array}$ \\
\hline Meaning & $\begin{array}{c}\text { The lack of } \\
\text { daily bowel } \\
\text { movements } \\
\text { can mean } \\
\text { Blood } \\
\text { deficiency. }\end{array}$ & $\begin{array}{c}\text { This could be a } \\
\text { symptom of Qi } \\
\text { deficiency. }\end{array}$ & $\begin{array}{c}\text { Commonly, this is a sign of } \\
\text { Yang deficiency. }\end{array}$ & $\begin{array}{c}\text { Commonly } \\
\text { a sign of Yin } \\
\text { deficiency. }\end{array}$ & Commonly this is a sign of Heat retention. \\
\hline
\end{tabular}

Therefore, the postpartum is considered to be one of the major moments of a woman life, regarding the health of the body. Not taking good care of the body in this period, not resting and not empower the body in this period can bring serious consequences for the rest of that woman's life. The energy issues developed in this period may take years to appear; only showing symptoms during menopause and aging, such as urinary incontinence [16].

The first 40 days are a time for rest, recovery and seclusion. The only function a woman should perform is to take care and feed her baby. After the birth Yin and Yang are unbalanced because woman uses much of her Yang energy in the birth. She has also lost Blood and Qi. She is considered to be internally cooler. Her Yang must be supported and no cold is allowed near a postpartum woman [16].

When these energies are not replaced by rest and good alimentation, according to the energy disturbances she is suffering, these may be one of the causes of energy deficiencies responsible for urinary incontinence [16].

However, women are not the only ones to have this problem, and the problem is not only associated with childbirth. According to TCM, for women and men the main cause of urinary incontinence is the Spleen and Kidney deficiency, and both deficiencies can be corrected with:

\section{Chinese dietary therapy}

\section{Acupuncture}

\section{Moxibustion}

4. Homeopathy, according to the theory developed by the author, entitled Constitutional Homeopathy of the Five Elements based on Traditional Chinese Medicine [17]

\section{Crystal-based medication.}

During this period, mothers are encouraged to reinvigorate their Yang by sipping herbal teas and eating warm, soft, easily digestible substances that serve to strengthen the Spleen, nourish Blood and Yin (especially for producing breast milk), and of course build Qi. Ideal foods include soups, bone broths, congee, cooked vegetables, and animal protein (if vegetarian, ensuring an abundance of plant-based protein and iron from legumes, tofu/tempeh, whole grains (quinoa, oatmeal), plus nuts and seeds [16].

\section{Diagnosis and main causes of energy imbalances}

Diagnosis: In order to make an energy imbalance, the patient is submitted to the questions proposed in [18-24] (Table 1).

Main Causes of Energy Imbalances: The main cause of energy imbalances were already extensively discussed in other articles of 


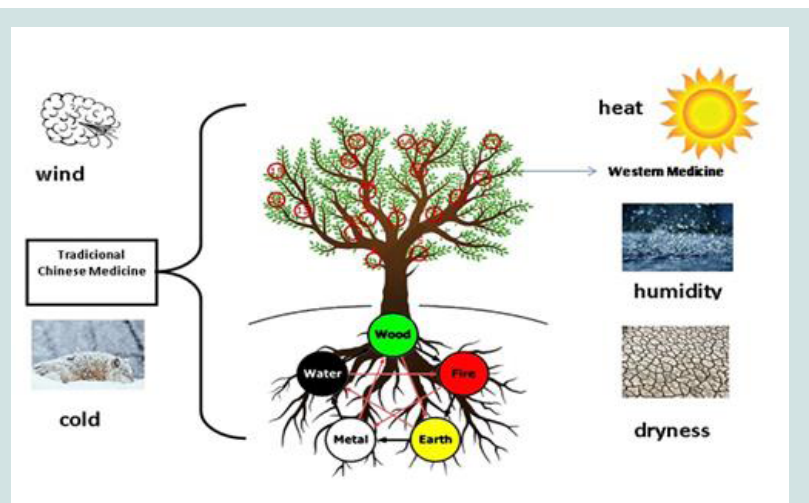

Figure 3: The metaphor of the Tree.

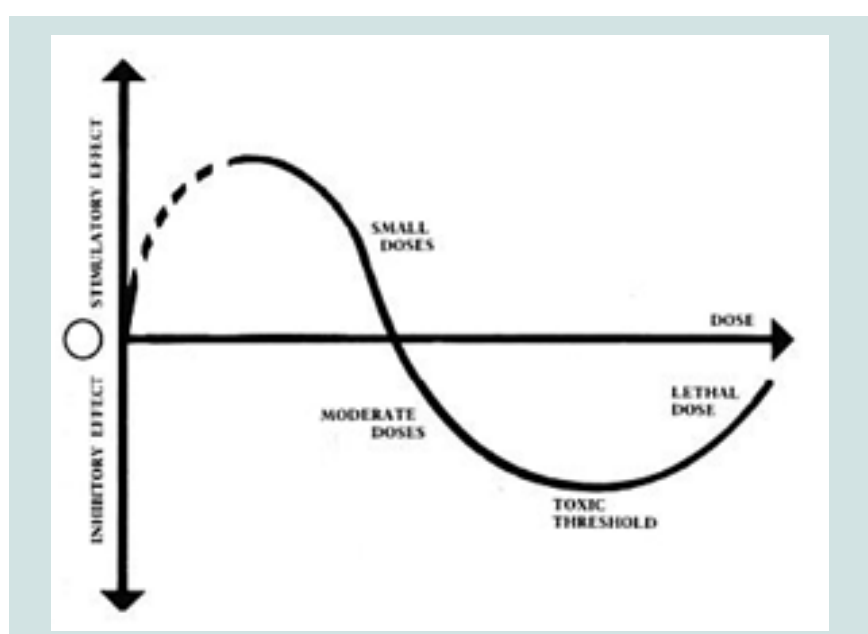

Figure 4: Arndt-Schultz Law.

the author. They are mainly related to diet, emotional habits, and exposure to certain external factors (Wind, Cold, Heat, Humidity and Dryness) [14,15].

Regarding diet, according to traditional Chinese medicine, foods will have their own energy as well. Some diseases can be easily cured only making diet changes. As Hippocrates stated: "Make your food your medicine, and your medicine your food". This sentence emphasizes the importance of a correct diet to maintain the health of the body, and to even cure diseases. As there are foods causing energy imbalances, there are also diets that can rebalance and restore the health of the body $[14,15]$.

Foods that cause Spleen-pancreas deficiency are very common in Western eating habits, such as ingestion of cold water, raw foods, dairy products and excessive sugar [25]. Foods that cause internal Heat build-up should be avoided. They are: fried foods, eggs, chocolate, honey, coconut, alcoholic beverage, dairy products especially when the cheese is melted, etc. [25].

Regarding the emotions, emotional problems will also influence the body. Different organs are affected in different ways by different energies. The Lungs for example, are affected by grief or sadness. When feeling too sad, pathologies regarding the respiratory system may appear. Spleen pancreas is affected by excessive worry. The Heart is mostly affected by excessive joy. Liver is affected by anger. Kidney is affected by fear $[14,15]$.

Regarding external factors, many carries should be applied in order to avoid the entrance of these external energies in the body. When suffering from energy deficiencies, the body becomes more vulnerable to the influence of external energies, continuing the process of energy deficiencies, creating an unhealthy cycle $[14,15]$.

The main external factors which influence the body are: Heat, Dampness, Cold, Dryness and Wind, as showed in (Figure 3). This figure was already explained in other articles of the author, and here it is exposed only to show the influence of external pathogenic factors on the state of health and disease. In the same way the tree is exposed, the human body also is $[14,15]$.

Hippocrates also stated on his study, the importance and influence of external pathogenic factors on the state of health, as well as the texts of the Yellow Emperor's Essential of Medicine [14,15].

\section{Tools for treatment}

Chinese dietary nutrition: Besides from what is recommended in Western medicine regarding diet, considering calories, vitamins, etc., the orientation on Chinese dietary nutrition is based on the energy imbalances of the patient, overlapped with the energy of each food [25].

In the case of the patient described in the case report, she had a Kidney-Yang deficiency, which is a deficiency on the energy that warms the body. Because of that, the patient was recommended to stop eating raw food, as they usually have cold energy. This would include all the vegetables and fruits with exception of lemon, papaya and apples. All the other fruits have to boiled or baked before consumption. In order to neutralize the energy, to not worsen the internal Cold presented by the patient [25].

Regarding the water intake, the energy of the water is also cold and because of that, the patient was oriented to drink the water lukewarm, after boiling on the stove, keeping it on a thermic bottle, in order to help on the warming process of the body of the patient, improving the Yang deficiency the patient was presenting [25].

The process of entry of energy on the body happen through the nutrition absorption, or the Spleen-pancreas meridian. In order to improve the absorption of nutrient, the patient was recommended to avoid foods that would imbalance the Spleen-pancreas meridians, such as dairy products, raw food, cold water and sweets [25].

The other meridian that may be compromising the Kidney deficiency of the patient was the Liver meridian, the patient was also oriented to avoid foods that would harm the energy of the Liver meridian and Gall-bladder, such as honey, coconut, dairy products, especially when the cheese is melted and alcoholic beverages [25].

The patient was also oriented to avoid foods that would diminish the energy of the Kidney directly, such as soda, coffee and matte tea [25].

One example is that the patient on the case report observed that the intake of soda would worsen her symptoms of urinary incontinence, demonstrating the influence of the diet on the results 


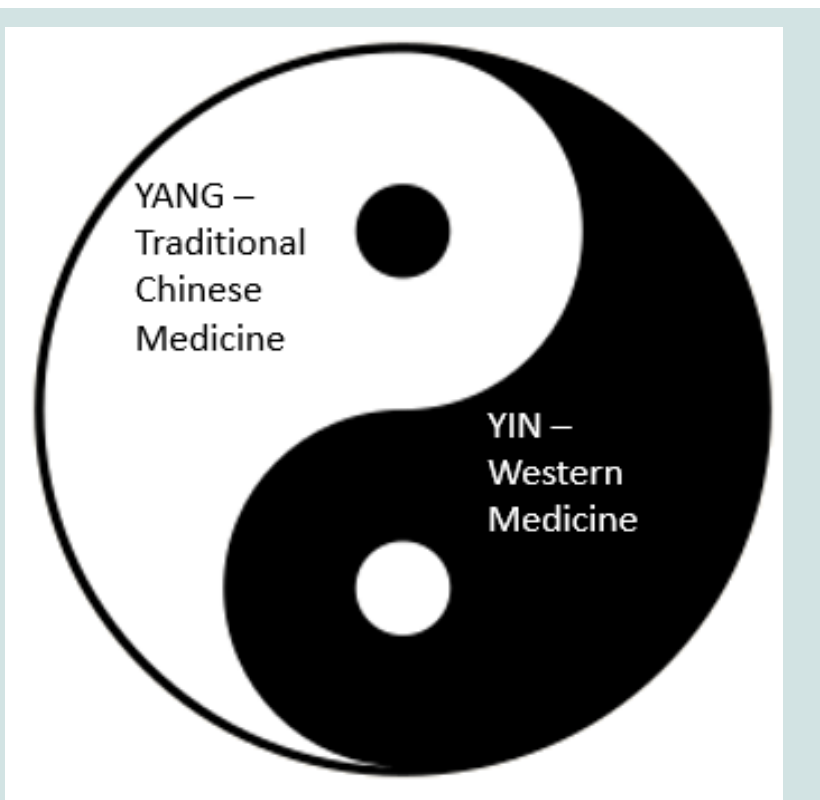

Figure 5: Traditional Chinese Medicine and Western Medicine - Yin and Yang Metaphor.

of the treatment [25].

Acupuncture and auricular acupuncture: Another important feature of traditional Chinese medicine is acupuncture. According to TCM, the diseases are associated with perturbations in the flow of $Q i$ energy. The goal of the acupuncture is to restore balance, correcting perturbations in the flow, using points close to the skin. Acupuncture is an important part of traditional Chinese medicine and from the Chinese health culture for more than 2,500 years [14,15].

Acupuncture is considered a medical specialty by the Federal Medical Council since 1995 [18-24].

Apex ear bloodletting is commonly linked to auricular acupuncture treatment, and according to TCM, it is believed to take off Heat retention [18-24].

Moxibustion: Moxibustion is a tool of traditional Chinese medicine. It is a form of heat therapy in which dried plant materials called "moxa" are burned on the skin or very near to the skin. According to traditional Chinese medicine, the moxibustion procedure has the ability of warming and invigorating the flow of Q $i$ in the body [26].

On the case of the patient described on the case report, she had Kidney-Yang deficiency, and felt very cold, especially on the extremities of the body. The main goal of the moxibustion on her case was to increase the Yang energy, so the patient would feel warmer after the sessions, meaning an improvement of her general energy state, and improving the Yang energy, responsible for containing urine, or prevent urinary incontinence.

\section{Arndt-Schultz Law}

According to the Arndt-Schultz's law, all drugs in high concentrations cause a reduction of vital energy, as demonstrated on (Figure 4). This reduction would generate the patient's energy weakness and the formation of internal Heat due to energy imbalances.
In the case of the drugs for urinary incontinence, it would not be different [18-24].

There is a need to have an integral view of the patient, deepening up to the level of his Yin and Yang energy, to understand, all the possible phenomena associated with the sick patient, including also the environment where he is inserted, which are the external factors that may start the imbalance [18-24].

Yin and Yang metaphor of Western and Traditional Chinese Medicine

In order to treat patients with urinary incontinence, it is important to comprehend the importance of the different medical perspectives. The body is not only physical and constituted by organs and tissues. It is also formed by energy. In Figure 5, a metaphor of Yin and Yang as the different kinds of medicine was created by the author. Nowadays, Western medicine and traditional Chinese medicine are opposite, but they could be complementary [18-24].

Traditional Chinese medicine can be considered as Yang energy, and Western medicine is considered Yin energy, already materialized. In the same way of the relationship of the Yin and Yang, traditional Chinese medicine starts where Western medicine ends, and viceversa. The relationship is not static, but dynamic [18-24].

\section{Conclusion}

The conclusion of this study is that it is possible to treat urinary incontinence clinically, without the necessity of using surgery.

To achieve this aim, it is important to understand the energy imbalances that are on the background of the symptoms of urinary incontinence, which in TCM, are related to Kidney-Yang deficiency. This deficiency is not static, but dynamic, and the treatment of other possible energy deficiencies (Yin, Yang, Qi and Blood), with changes on the dietary habits (using TCM reasoning), improving the energy of the patients with auricular acupuncture, moxibustion and replenishment of the chakras energy with homeopathy and crystalbased medication are possible tools to treat this kind of patient.

The patients that make surgery and present no improvement, or the symptoms return, may be because the energy imbalances were not treated. The association between the energy-based treatment and surgery may also be relevant in cases where the surgery is necessary, to avoid the relapse on the symptoms.

\section{References}

1. Aoki Y, Brown HW, Brubaker L, Cornu JN, Daly O, et al. (2017) Urinary incontinence in women. Nat Rev Dis Primers 3: 17042.

2. Luber KM (2004) The definition, prevalence, and risk factors for stress urinary incontinence. Rev Urol 6 Suppl 3: S3-S9.

3. Wang Y, Zhishun L, Peng W, Zhao J, Liu B (2013) Acupuncture for stress urinary incontinence in adults (Review). Cochrane Database Syst Rev 7: CD009408.

4. Kim Y, Chancellor MB (2004) Incontinence after Childbirth. Rev Urol 6: 48-49.

5. Biswas B, Bhattacharyya A, Dasgupta A, Karmakar A, Mallick N, et al. (2017) Urinary Incontinence, Its Risk Factors, and Quality of Life: A Study among Women Aged 50 Years and above in a Rural Health Facility of West Bengal. J Midlife Health 8: 130-136.

6. Nilsson CG, Kuuva N, Falconer C, Rezapour M, Ulmsten U (2001) Long-term 
results of the tension-free vaginal tape (TVT) procedure for surgical treatment of female stress urinary incontinence. Int Urogynecol J Pelvic Floor Dysfunct 12 Suppl: S5-S8.

7. Wilson L, Brown JS, Shin GP, Luc KO, Subak LL (2001) Annual direct cost of urinary incontinence. Obstet Gynecol 98: 398-406.

8. Herr HW (2008) 'I will not cut . . '? the oath that defined urology. Journal Compilation (c) 2008 BJU International 102: 769-772.

9. Khan HA, Baig FK, Mehboob R (2017) Nosocomial infections: Epidemiology prevention, control and surveillance. Asian Pacific J Tropical Biomedicine 7 478-482.

10. National Association for Continence. 2018 National Association for Continence. All rights reserved. Unauthorized use prohibited. NAFC is a qualified 501(c)(3) tax-exempt organization.

11. Xiu-Min L, Brown L (2009) Efficacy and mechanisms of action of traditiona Chinese medicines for treating asthma and allergy. J Allergy Clin Immuno 123: 297-308.

12. Zhong D, Tang W, Geng D, He C (2019) Efficacy and safety of acupuncture therapy for urinary incontinence in women: A systematic review and metaanalysis. Medicine (Baltimore) 98: e17320.

13. Yang Shi Lin, Li Zheng Hua. Pathmechanims of the Kidney. Paradigm, Taos, New Mexico.

14. Maoshing NI. The Yellow Empero's Classic of Medicine. Shambala, Boston and London.

15. Hua-Ching N, I-Ching I The Book of Changes and the Unchanging Truth Tao of Wellness.

16. Giovanni Maciocia. Obstetrícia e Ginecologia em Medicina Chinesa. Editora Roca.
17. Ling HW (2020) Constitutional Homeopathy of the Five Elements based on Traditional Chinese Medicine. Acta Sci Med Sci 4: 57-69.

18. Ling HW (2018) "Could Postsurgical Nosocomial Cellulitis be Treated without the Use of Antibiotics?". Acta Scientific Microbiology 1: 24-31.

19. Ling HW. Why Are Diabetic Patients Still Having Hyperglycemia despite Diet Regulation, Antiglycemic Medication and Insulin? Int J Diabetes Metabolic Dis.

20. Ling HW (2019) "Can Biomaterial Surgical Implants Influence the Body's Health?". Acta Sci Med Sci 3: 62-71.

21. Huang WL (2018) Why do patients still catch hospital infections despite the practice of infection prevention and control programs? Acta Scientific Microbiology 1: 34-43.

22. Ling HW (2019) Can we treat atopic dermatitis without using corticosteroids? J Pediat Infants 2: 08-19.

23. Huang WL (2018) The treatment of asthma based on traditional Chinese medicine and homeopathy. J Pediat Infants 1: 24-30.

24. Ling HW (2019) Is it Possible to Treat Community-Acquired and Nosocomia Infections with the Same Method, Without the Use of Antibiotics? J Appl Microb Res 2: 01-13.

25. Perini M. Terapia Dietética Chinesa. Editora Loyola pp: 240

26. Hye-Yoon L, Young-Ju Y, Jun-Yong C, Jin-Woo H, Lee I, et al. (2018) Effectiveness and safety of moxibustion for alleviating symptoms of overactive bladder: A prospective, randomized controlled, crossover-design, pilot study. Randomized Controlled Trial Medicine (Baltimore) 97: e12016. 